\title{
Diagnostic confirmation of amoxicillin allergy in children treated at the Division of Pediatric Allergy
}

\author{
Christian Morelo Torres, M.D. ${ }^{a}$, Alfredo Eymann, M.D. ${ }^{b}$, Natalia Petriz, M.D. ${ }^{a}$ and Claudio A. Parisi, M.D. ${ }^{a}$
}

\begin{abstract}
Amoxicillin is a beta-lactam antibiotic commonly indicated in pediatrics and the most frequent cause of drug allergies. Objectives. To determine the proportion of confirmed amoxicillin allergy in children with diagnostic suspicion seen at the Division of Pediatric Allergy.

Population and methods. This descriptive, retrospective study was done between January 2009 and January 2017 in children younger than 18 years with diagnostic suspicion of amoxicillin allergy. The diagnosis was based on questions and specific tests. Results. A total of 234 patients were included; amoxicillin allergy was diagnosed in $10.7 \%$ (95\% confidence interval: 7-15). These patients had a higher prevalence of immediate symptoms (40\% vs. $22 \%, p=0.048)$ and prior exposure to beta-lactams ( $84 \%$ vs. $56 \%, p=0.007)$.

Conclusion. Amoxicillin allergy in children referred to specialists was confirmed in $10.7 \%$.

Key words: hypersensitivity, drugs, beta-lactams, children, epidemiology.
\end{abstract}

http: / / dx.doi.org/10.5546/ aap.2020.eng.47

To cite: Morelo Torres C, Eymann A, Petriz N, Parisi CA. Diagnostic confirmation of amoxicillin allergy in children treated at the Division of Pediatric Allergy. Arch Argent Pediatr 2020;118(1):47-51. a. Division of Pediatric Allergy.

b. Department of Clinical Pediatrics.

Hospital Italiano de Buenos Aires.

E-mail address:

Claudio A. Parisi, M.D.: claudio.parisi@hiba.org.ar

Funding: None.

Conflict of interest: None.

Received: 2-28-2019

Accepted: 6-17-2019

\section{INTRODUCTION}

Allergic drug reactions (ADRs) are classified based on the speed of symptom onset, into immediate and non-immediate. ${ }^{1,2}$ Immediate ADRs may go from symptoms limited to the skin to severe and life-threatening reactions. ${ }^{2}$ Non-immediate ADRs occur as of one hour after taking the antibiotic; most are mild and include maculopapular rash;, ${ }^{2,3}$ and may rarely lead to more severe conditions. ${ }^{1}$

Amoxicillin is the most common cause of ADRs. ${ }^{4}$ Approximately $70 \%$ of patients with viral infections receive empiric antibiotic treatment with amoxicillin while maculopapular rash secondary to this condition is usually wrongly considered an ADR. ${ }^{3,5}$

In spite of this, diagnosis is usually not confirmed in most patients. As per our knowledge, there are scarce epidemiological data about this condition in Argentine children. The objective of this study was to determine the rate of confirmed amoxicillin allergy in children with diagnostic suspicion seen at the Division of Pediatric Allergy.

\section{POPULATION AND METHODS}

This was a descriptive, retrospective study carried out between January $1^{\text {st }}, 2008$ and January $1^{\text {st }}, 2017$.

The population was made up of patients younger than 18 years whose electronic medical record (EMR) included the following: amoxicillin allergy, suspected amoxicillin allergy, beta-lactam allergy or suspected beta-lactam allergy, and who had attended at least one office visit to the Division of Pediatric Allergy.

Exclusion criteria included patients with incomplete data, allergy to a different beta-lactam antibiotic, absence of specific diagnostic tests, and severe non-immediate reactions, such as StevensJohnson syndrome, toxic epidermal necrolysis, and drug rash with eosinophilia and systemic symptoms. ${ }^{1}$

The following outcome measures were analyzed: age, sex, personal and close family history of allergy (asthma, allergic rhinitis, atopic 
dermatitis, food allergy, drug allergy and/or insect bite allergy), prior exposure to amoxicillin and/or beta-lactam antibiotics.

The following diagnostic tests were done:

Serum-specific immunoglobulin E (IgE): IgE levels were measured for amoxicillin, penicillin $G$ and $V$, and ampicillin using an enzyme-linked immunosorbent assay (ELISA). Values above $0.36 \mathrm{IU} / \mathrm{mL}$ were considered positive.

Skin prick test (SPT): 6 Amoxicillin was used for the SPT (level: $25 \mathrm{mg} / \mathrm{mL}$ ). ${ }^{6}$ A wheal $3 \mathrm{~mm}$ greater than the negative control was considered positive.

Intradermal reaction (IDR) skin test: This was done using the Mantoux technique with amoxicillin (level: $25 \mathrm{mg} / \mathrm{mL}$ ). ${ }^{6}$ A wheal with erythema and a diameter $\geq 3 \mathrm{~mm}$ compared to the negative control was considered positive. ${ }^{6}$ In Argentina, the major determinant, benzylpenicilloyl polylysine and minor determinant mixtures ${ }^{4}$ are not available, so these were not tested.

Atopy patch test (APT): A pure test substance dilution $^{7}$ was applied under occlusion into Finn chambers in the interscapular area for 2 days; readings were made at 48 and $96 \mathrm{~h}$. Positive tests were determined based on the International Contact Dermatitis Research Group scoring system. ${ }^{8}$

Controlled challenge test (CCT): Amoxicillin was administered orally at a dose of $80 \mathrm{mg} /$ $\mathrm{kg} /$ day in the hospital setting, split into three doses 20 minutes apart. Patients were considered allergic if, during the CCT, they had immediate hypersensitivity symptoms, i.e., a positive test. Patients without suspicious symptoms continued taking the antibiotic for 5 days and remained in contact with the health care team to report any potential, non-immediate symptom as per the protocol modified by Mori et al. ${ }^{3}$

Amoxicillin allergy was defined as the presence of immediate symptoms (anaphylaxis, angioedema, urticaria, bronchospasm) and / or the presence of non-immediate symptoms (maculopapular, pustular, bullous eczema or rash $)^{9}$ with at least one positive confirmatory test.

Patients were considered to have a high risk for allergy if they were older than 12 years of age and/or had a history of severe events (visit to the emergency department, respiratory distress symptoms and / or adrenaline use).${ }^{10} \mathrm{In}$ these patients, if the ELISA test was negative, an SPT and a IDR skin test were done; patients considered to have a low risk had a challenge test done immediately after the negative ELISA test.

An APT was done in all patients with delayed reactions. An ELISA test was done in all patients, regardless of the mechanism involved. The diagnostic process is described in Figure 1.

This study was approved by the site's Ethics Committee for Research Protocols.

\section{Statistical analysis}

Quantitative outcome measures were expressed as mean and standard deviation (SD), whereas categorical outcome measures, as frequency and percentage. The proportion of confirmed amoxicillin allergy was estimated based on the number of patients with confirmed amoxicillin allergy diagnosis as the numerator and the total number of patients referred to the outpatient office or who attended the Division of Pediatric Allergy spontaneously due to suspected amoxicillin allergy as the denominator. The differences among continuous outcome measures were analyzed using a $t$ test for independent samples, while categorical outcome measure proportions were analyzed using a $\chi^{2}$ test. Statistically significant outcome measures in the univariate analysis were included in the multivariate model. A value of $p<0.05$ was considered significant. The statistical software package used was STATA 13.

\section{RESULTS}

A total of 234 patients were included in the study (Figure 2); $52 \%$ were males; and the patients' mean age was 3.6 years (SD: 3.1 years). Among total patients, 178 (76\%) showed delayed symptoms and $56(24 \%)$, immediate symptoms (Table 1); 138 (59 \%) had been previously exposed to a beta-lactam antibiotic at least once.

Amoxicillin allergy was diagnosed in 25 patients, which accounted for $10.7 \%$ (95\% confidence interval [CI]: 7-15) of the study population (Figure 2). Diagnosis was confirmed by ELISA, skin tests or CCT in $9(36 \%), 3(12 \%)$, and $13(52 \%)$ children, respectively.

In the univariate analysis, patients with confirmed amoxicillin allergy had a higher prevalence of immediate symptoms ( $40 \%$ vs. $22 \%, p=0.048)$ and prior exposure to betalactams ( $84 \%$ vs. $56 \%, p=0.007)$. No differences were observed in other outcome measures (Table 2).

In the multivariate analysis, the presence of immediate symptoms and prior exposure to betalactams remained statistically significant. The 


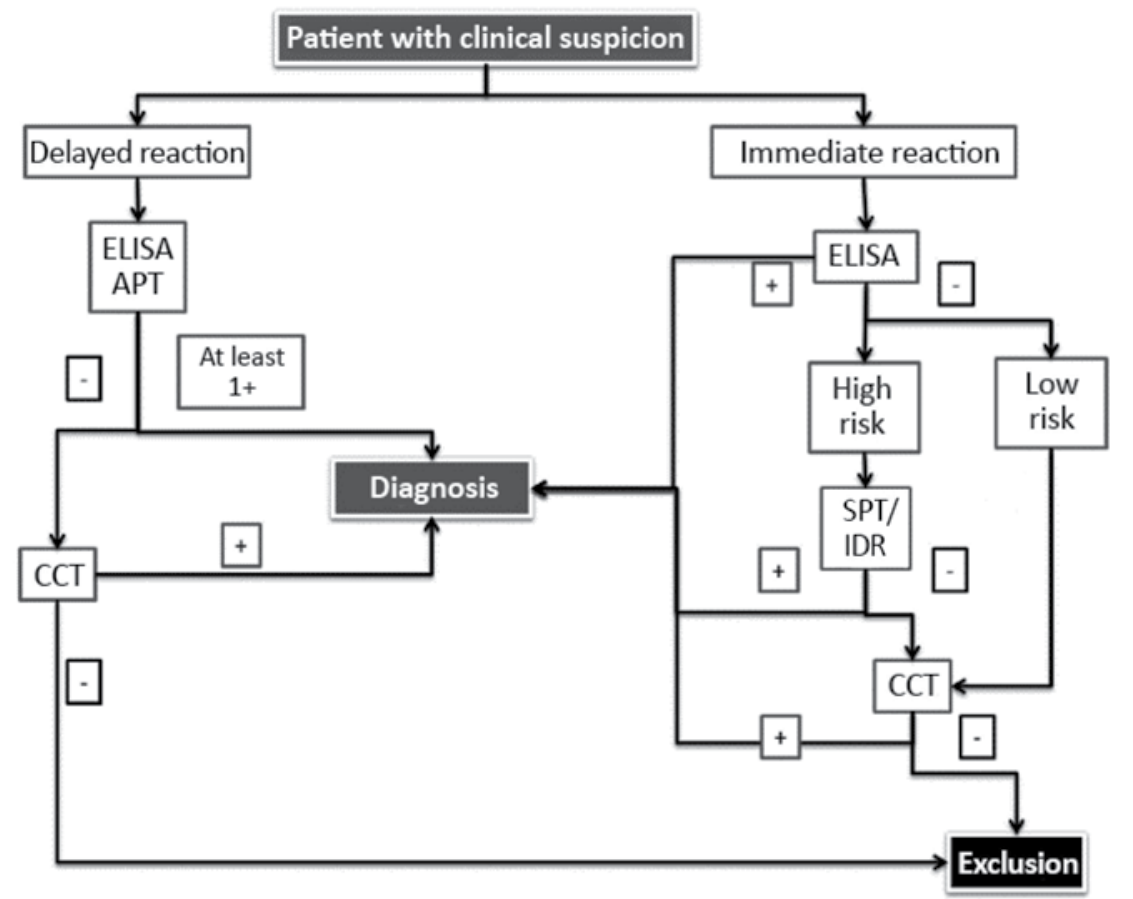

APT: atopy patch test; CCT: controlled challenge test; SPT: skin prick test;

IDR: intradermal reaction; PPD: purified protein derivative skin test.

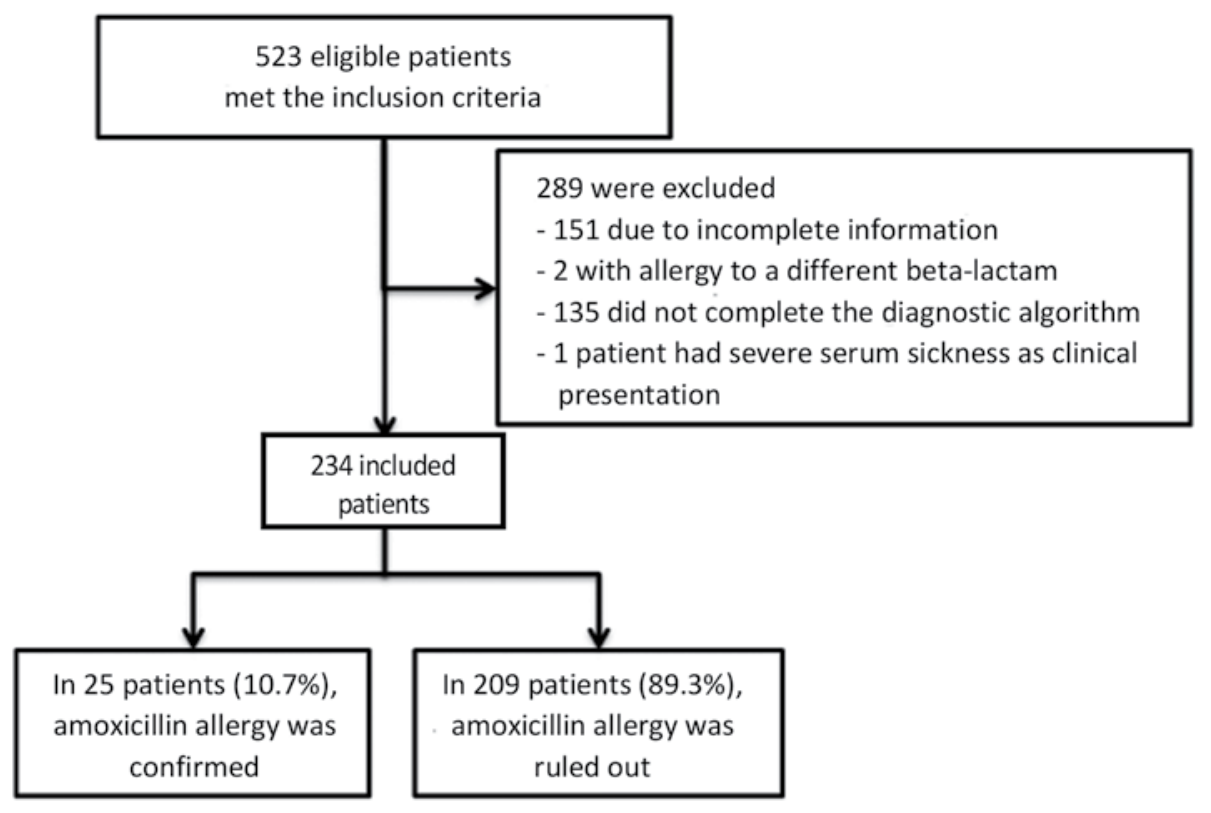


odds ratio (OR) for confirmed amoxicillin allergy in children with immediate symptoms was 2.45 (95\% CI: 1.01-5.87, $p=0.049$ ). In turn, the OR for confirmed amoxicillin allergy in children with prior beta-lactam use was 4.24 (95\% CI: 1.3912.90, $p=0.011$ ).

\section{DISCUSSION}

Although the rate of suspected amoxicillin allergy is high, a large percentage of studied children are not allergic. ${ }^{11,12}$ In this study, amoxicillin allergy was confirmed in $10.7 \%$ of children referred to a specialist, similar to what has been reported by Mori et al., ${ }^{3}$ who identified $9.6 \%$ of 200 Italian children using a similar study methodology.

Children with immediate symptoms were 1.5 times more likely to have an ADR than those with delayed symptoms, and those with prior exposure to beta-lactam antibiotics showed a 3.2 times stronger association with confirmed amoxicillin allergy diagnosis.

No significant relation was observed with a personal and family history of allergy, as in the study by Ponvert et al., ${ }^{13}$ but unlike the one by Faitelson et al. ${ }^{10}$

Few diagnostic confirmation studies have been published and, in general, amoxicillin allergy is over-diagnosed. ${ }^{14}$ This study approached a problem for which few data are available and

TABLE 1. Clinical characteristics of patients with suspected amoxicillin allergy, $n=234$

\begin{tabular}{|c|c|c|c|c|}
\hline & & & $\mathbf{n}$ & $\%$ \\
\hline \multirow[t]{2}{*}{ Sex } & Female & & 114 & 48.7 \\
\hline & Male & & 120 & 51.3 \\
\hline \multirow{5}{*}{ Type of symptoms } & Immediate & Skin (urticaria/angioedema, maculopapular rash) & 52 & 22.2 \\
\hline & & Gastrointestinal & 1 & 0.4 \\
\hline & & Anaphylaxis & 3 & 1.3 \\
\hline & Delayed & Maculopapular rash & 128 & 54.7 \\
\hline & & Urticaria & 50 & 21.4 \\
\hline \multirow[t]{2}{*}{ Prior exposure to beta-lactams } & Yes & & 138 & 59 \\
\hline & No & & 96 & 41 \\
\hline \multirow[t]{5}{*}{ Personal history of allergy } & Yes & Asthma-bronchospasm & 37 & 50.7 \\
\hline & & Rhinitis & 30 & 41 \\
\hline & & Atopic dermatitis & 9 & 12.3 \\
\hline & & Other & 10 & 14 \\
\hline & No & & 161 & 69 \\
\hline \multirow[t]{2}{*}{ Family history of allergy } & Yes & & 112 & 48 \\
\hline & No & & 112 & 52 \\
\hline
\end{tabular}

TABLE 2. Comparison of patients with amoxicillin allergy

\begin{tabular}{|c|c|c|c|c|}
\hline & & $\begin{array}{l}\text { Without allergy } \\
(\mathrm{n}=209) \\
\% \\
\end{array}$ & $\begin{array}{c}\text { With allergy } \\
(\mathbf{n}=25) \\
\% \\
\end{array}$ & ${ }^{*} p$ value \\
\hline *Sex & $\begin{array}{l}\text { Female } \\
\text { Male }\end{array}$ & $\begin{array}{l}48 \\
52\end{array}$ & $\begin{array}{l}52 \\
48\end{array}$ & 0.711 \\
\hline **Age in years & $($ mean $\pm \mathrm{SD})$ & $3.48 \pm 3.20$ & $3.92 \pm 2.94$ & 0.495 \\
\hline *Immediate symptoms & $\begin{array}{l}\text { Yes } \\
\text { No }\end{array}$ & $\begin{array}{l}22 \\
88\end{array}$ & $\begin{array}{l}40 \\
60\end{array}$ & $\begin{array}{l}0.048 \\
0.048\end{array}$ \\
\hline${ }^{*}$ Prior exposure to beta-lactams & $\begin{array}{l}\text { Yes } \\
\text { No }\end{array}$ & $\begin{array}{l}56 \\
44\end{array}$ & $\begin{array}{l}84 \\
26\end{array}$ & 0.007 \\
\hline *Personal history of allergy & $\begin{array}{l}\text { Yes } \\
\text { No }\end{array}$ & $\begin{array}{l}30 \\
70\end{array}$ & $\begin{array}{l}44 \\
66\end{array}$ & 0.148 \\
\hline${ }^{*}$ Family history of allergy & $\begin{array}{l}\text { Yes } \\
\text { No }\end{array}$ & $\begin{array}{l}45 \\
55\end{array}$ & $\begin{array}{l}60 \\
40\end{array}$ & 0.206 \\
\hline
\end{tabular}

${ }^{*} \chi^{2}$ test. ${ }^{* *}$ Student's t test. SD: standard deviation. 
underlines the importance of patient questioning and diagnostic tests to prevent the negative impact of classifying patients with a non-existent condition.

This study has some limitations: its retrospective design; out of the total sample, $44.7 \%$ met the inclusion criteria; a single participating site; and that study children had been referred to a specialist, so it may be inferred that the prevalence in the general pediatric population may be lower.

To conclude, in our setting, more than half of children who see a specialist due to suspected amoxicillin allergy are not allergic.

\section{Acknowledgments}

We would like to thank Carolina Antonietti, M.D., for her major collaboration in this study.

\section{REFERENCES}

1. Romano A, Warrington R. Antibiotic allergy. Immunol Allergy Clin North Am. 2014; 34(3):489-506.

2. Pavlos R, Mallal S, Ostrov D, Buus S, et al. T Cell-mediated hypersensitivity reactions to drugs. Annu Rev Med. 2015; 66:439-54.

3. Mori F, Cianferoni A, Barni S, Pucci N, et al. Amoxicillin Allergy in children: five-day provocation test in the diagnosis of nonimmediate reactions. J Allergy Clin Immunol Pract.
2015; 3(3):375-80.e1.

4. Comité Nacional de Alergia. Reacciones alérgicas a betalactámicos en pediatría: recomendaciones para su diagnóstico y tratamiento. Arch Argent Pediatr. 2019; 117(Suppl 1):S24-36

5. Jappe U. Amoxicillin-induced exanthema in patients with infectious mononucleosis: allergy or transient immunostimulation? Allergy. 2007; 62(12):1474-5.

6. Torres MJ, Blanca M, Fernández J, RomanoA, etal. Diagnosis of immediate allergic reactions to beta-lactam antibiotics. Allergy. 2003; 58(10):961-72.

7. DeGroot AC. Patch Testing. Test concentration and vehicles for 3700 chemicals. $2^{\text {nd }}$ ed. New York: Elsevier; 1994.

8. Fischer T, Maibach HI. Patch Testing in allergic contact dermatitis. Sem Dermatol. 1986; 5:59-68.

9. Demoly P, Romano A. Update on Beta-lactam Allergy diagnosis. Curr Allergy Asthma Rep. 2005; 5(1):9-14.

10. Faitelson Y, Boaz M, Dalal I. Asthma, Family History of Drug Allergy, and Age Predict Amoxicillin Allergy in Children. J Allergy Clin Immunol Pract. 2018; 6(4):1363-7.

11. Impicciatore P, Choonara I, Clarkson A, Provasi D, et al. Incidence of adverse drug reactions in paediatric in/ out-patients: a systematic review and meta-analysis of prospective studies. Br J Clin Pharmacol. 2001; 52(1):77-83.

12. Romano A. Recognising antibacterial hypersensitivity in children. Paediatr Drugs. 2000; 2(2):101-12.

13. Ponvert C, Perrin Y, Bados-Albiero A, Le Bourgeois M, et al. Allergy to betalactam antibiotics in children: results of a 20-year study based on clinical history, skin and challenge tests. Pediatr Allergy Immunol. 2011; 22(4):411-8.

14. Erkoçoglu M, Kaya A, Civelek E, Ozcan C, et al. Prevalence of confirmed immediate type drug hypersensitivity reactions among school children. Pediatr Allergy Immunol. 2013; 24(2):160-7. 